\title{
Caveolin-I interacts with the Gag precursor of murine leukaemia virus and modulates virus production Zheng $\mathrm{Yu}^{\dagger 1}$, Christiane Beer ${ }^{\dagger 1,2}$, Mario Koester ${ }^{1}$ and Manfred Wirth*1
}

Address: ${ }^{1}$ Molecular Biotechnology Division, German Research Centre for Biotechnology, GBF, Mascheroder Weg 1, Braunschweig, Germany and ${ }^{2}$ Department of Molecular Biology, Aarhus University, C.F. Mollers Alle 130, Aarhus, Denmark

Email: Zheng Yu - zyu@gbf.de; Christiane Beer - chb@mb.au.dk; Mario Koester - mks@gbf.de; Manfred Wirth* - mwi@gbf.de

* Corresponding author †Equal contributors

Published: 06 September 2006

Virology Journal 2006, 3:73 doi:10.1186/1743-422X-3-73

This article is available from: http://www.virologyj.com/content/3/I/73

(c) 2006 Yu et al; licensee BioMed Central Ltd.

This is an Open Access article distributed under the terms of the Creative Commons Attribution License (http://creativecommons.org/licenses/by/2.0), which permits unrestricted use, distribution, and reproduction in any medium, provided the original work is properly cited.
Received: 02 June 2006

Accepted: 06 September 2006

\begin{abstract}
Background: Retroviral Gag determines virus assembly at the plasma membrane and the formation of virus-like particles in intracellular multivesicular bodies. Thereby, retroviruses exploit by interaction with cellular partners the cellular machineries for vesicular transport in various ways.
\end{abstract}

Results: The retroviral Gag precursor protein drives assembly of murine leukaemia viruses (MLV) at the plasma membrane (PM) and the formation of virus like particles in multivesicular bodies (MVBs). In our study we show that caveolin-I (Cav-I), a multifunctional membrane-associated protein, co-localizes with Gag in a punctate pattern at the PM of infected NIH 3 T3 cells. We provide evidence that Cav-I interacts with the matrix protein (MA) of the Gag precursor. This interaction is mediated by a Cav-I binding domain (CBD) within the N-terminus of MA. Interestingly, the CBD motif identified within MA is highly conserved among most other $\gamma$ retroviruses. Furthermore, Cav-I is incorporated into MLV released from NIH 3 T3 cells. Overexpression of a GFP fusion protein containing the putative CBD of the retroviral MA resulted in a considerable decrease in production of infectious retrovirus. Moreover, expression of a dominant-negative Cav-I mutant affected retroviral titres significantly.

Conclusion: This study demonstrates that Cav-I interacts with MLV Gag, co-localizes with Gag at the PM and affects the production of infectious virus. The results strongly suggest a role for Cav$I$ in the process of virus assembly.

\section{Background}

The Gag protein precursor is a polyprotein consisting of matrix protein (MA), protein p12, capsid protein (CA) and nucleocapsid protein (NC) and represents a principal actor in retrovirus assembly at the plasma membrane (PM). The Gag precursor is synthesized on free ribosomes and myristoylated at glycin 2 in MA. Fatty acylation is sufficient to localize Gag at the plasma membrane, where in the presence of the envelope (Env) proteins viral particles assemble. In the final stage of budding a membrane fis- sion event is required for efficient separation of newly synthesized retroviruses. Concurrent with budding, the Gag polyprotein is cleaved by the retroviral protease into MA, $\mathrm{CA}, \mathrm{NC}$ and other virus-specific Gag derived proteins. Distinct regions in the Gag protein were identified which mediate membrane binding, multimerization and induce separation of nascent virus particles from the cell [1].

Gag alone is sufficient to induce the formation of viruslike particles (VLPs) [1]. The formation of infectious par- 
ticles, however, requires co-localization of Env and Gag and occurs in a cell-dependent manner either at the plasma membrane or at internal membranes. Mutational analysis of Gag of certain retroviruses defined several regions important for Gag transport and efficient membrane anchoring, as mutant viruses were blocked in membrane association or redirected to multivesicular bodies (MVBs) localized in the cytoplasm. It has been shown, that myristoylation of Pr65 gag at Gly2 at the aminoterminus of the viral MA is substantial for Moloney murine leukaemia virus (MoMLV) particle formation and budding $[2,3]$, and is required for efficient binding to the plasma membrane. In addition, a run of basic residues or a cluster of lipophilic amino acids close to the aminoterminus are involved in Gag transport to the site of virus assembly [48] However, the requirement for fatty acylation can be overcome by other molecules. Thus, protein-protein interactions have been postulated to be necessary for efficient protein localization in lipid rafts [9].

Surprisingly, Gag transport turned out to be a complex process involving several cellular proteins. Early experiments with MLV and Rous sarcoma virus (RSV) demonstrated that deletion of a region located between MA and CA affected virus assembly. The same was true for human immunodeficiency virus (HIV) when a region at the carboxy-terminus of the Gag precursor was deleted. These early notions led to the identification of $\mathrm{L}$-domains which recruit the cellular machinery for intravesicular transport of Gag [reviewed in 10,11]. The identified L-domains differ in their sequence within the retrovirus family and each retroviral L-domain binds specific factors to redirect Gag into the MVB pathway, thereby directing the budding and egress of virions. The subject is still puzzling, as viral Gag proteins contain several interacting motifs and their importance varies in different retroviruses [12].

Formation of infectious MLV as well as successful pseudotyping of MLV vectors requires Env co-colocalization with Gag $[13,14]$. Moreover, incorporation of cellular membrane proteins into virions seems to be dependent on colocalization. Interestingly, the incorporation reflects the cell type, intracellular transport and the platform of assembly and budding. Lipid rafts have been suggested as portals for retrovirus exit. Env localization in lipid rafts has been demonstrated for ecotropic MoMLV [15] as well as for amphotropic 4070A MLV more recently [16].

When studying 4070A Env localization in infected NIH3T3 cells, we noticed co-localization of Env with Cav1, a multi-functional membrane protein. Cav-1 is present in lipid rafts and its oligomerization leads to caveolae formation. Caveolae are the main actors for a clathrin-independent endocytic pathway, first identified for internalization of GPI anchored proteins [17]. Moreover,
Cav-1 functions as scaffolding protein to organize and concentrate a growing list of proteins involved in diverse signaling processes. Finally, Cav-1 is involved in cholesterol transport [18].

We wondered whether the co-localization of Cav-1 and 4070A Env in the PM of mouse NIH3T3 cells results in release of 4070A MLV (referred to as A-MLV) containing Cav-1. Here, we proved the presence of considerable amounts of Cav-1 within A-MLV as well as MoMLV. Furthermore, MLV Gag co-localizes with Cav-1 at the PM. Coimmuno-precipitations revealed that both proteins interact presumably via a caveolin-binding domain (CBD) within the aminoterminal region of MA. The CBD is highly conserved in Gag of most $\gamma$-retroviruses and competition experiments using CBD fusion proteins or a Cav1 dominant-negative mutant revealed that Gag-Cav-1 interaction modulates MLV production.

\section{Results \\ Amphotropic and ecotropic murine leukemia virions incorporate Caveolin-I}

Recently, we reported co-localization of Cav- 1 and Env of A-MLV [16] and presented hints for Cav-1 incorporation into A-MLV released from mouse NIH 3T3 cells [19]. To confirm our initial results and to extend our findings to the ecotropic MoMLV we investigated whether Cav- 1 is included into released virions. For that purpose we analysed A-MLV and MoMLV propagated in NIH 3 T3 cells. Viruses were purified by ultracentrifugation followed by sucrose gradient centrifugation. Viral proteins were separated by SDS-PAGE and analysed by Western Blot using anti-Cav-1 antibodies. Figure 1 demonstrates that Cav- 1 is incorporated into MoMLV (lane 3) as well as A-MLV (lane 4). A signal with the size expected for Cav-1 isoforms (21$24 \mathrm{kD}$ ) could be detected in processed virus samples (lane $3,4)$ which comigrates with positive control samples from cell lysates (lane 1,2). Processed supernatants of mockinfected non-virusproducing NIH 3T3 cells did not give rise to a signal (data not shown, [19]). Therefore, both MLV strains incorporate Cav-1 into the viral membrane, presumably during the process of budding from lipid rafts of NIH 3T3 cells.

\section{Co-localization of MLV-Gag and caveolin- I}

To address the question whether MLV Gag and Cav-1 colocalize, we investigated NIH 3T3 and 293 cells transfected with expression plasmids carrying Gag or Cav-1 fused C-terminally to fluorescent proteins (FPs) using confocal microscopy. It has been shown that attachment of GFP to the C-terminus of Cav-1 does not interfere with its localization, fatty acylation or oligomerization properties [20-22]. Similarly, C-terminal fusions of FPs to Gag have been proven to be valuable tools to study localization, incorporation and budding of MLV Gag [23,24]. 


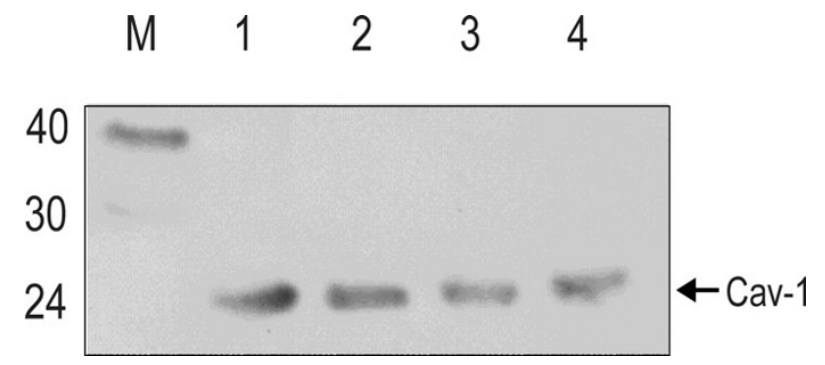

Figure I

Caveolin-I is incorporated into MLV virions. Ecotropic MLV and amphotropic MLV were pelleted from supernatants of infected NIH3T3 cells and purified by sucrose gradient centrifugation and virions were lysed and subjected to SDSPAGE (10\%) followed by Western blot analysis using rabbit anti Cav-I as primary antibody. Lane I: Cav-I positive control, human carcinoma cell lysate; Lane 2: Cav-I positive control, NIH 3T3 cell lysate. Lane3: Ecotropic MLV Lane 4: Amphotropic MLV. M Marker, molecular weight in kDa.

NIH 3T3 cell transfected with Cav-1-GFP, showed a typical surface staining and exhibited the expected expression pattern, but also several scattered spots distributed throughout the cytoplasm and a prominent accumulation of Cav-1 positive membranes at the center of the cell in perinuclear regions were observed (Fig. 2A). Gag-RFP appeared in a punctate pattern distributed allover the cytoplasm, accumulated in membranes in perinuclear regions and at or close to the PM like beads on a string (Fig. 2A and 2B). In 293 cells, which contain less endogeneous Cav-1 than NIH 3T3 cells [25] Cav-1-GFP as well as Gag-RFP were concentrated in patches at the edge of the cell, however, Cav-1-GFP accumulated predominantly in a perinuclear region in the center of the cell. Gag-RFP vesicles were also concentrated in this region, however, to a lesser extent (Data not shown). In cotransfected NIH 3T3 cells Gag-RFP and Cav-1-GFP spots co-localized to a certain extent at the PM and in perinuclear regions, but to a much lesser extent in the cytoplasm (Fig. 2A). Interestingly, when infected cells were used for the transfection experiments the Cav-1-GFP and Gag-RFP fluorescence patterns did not change considerably (see Additional file 1). However, in certain cases, Cav-1 predominantly stacked in perinuclear regions, while the pattern of GagRFP remained unaltered (data not shown).

In the experiments described endogenous Cav-1, even in low levels, may compete with Cav-1-GFP expression. Moreover, overexpression of Cav-1-GFP may favour stacking of the GFP fusion protein in perinuclear regions. Therefore, we repeated the experiments using Gag-RFP

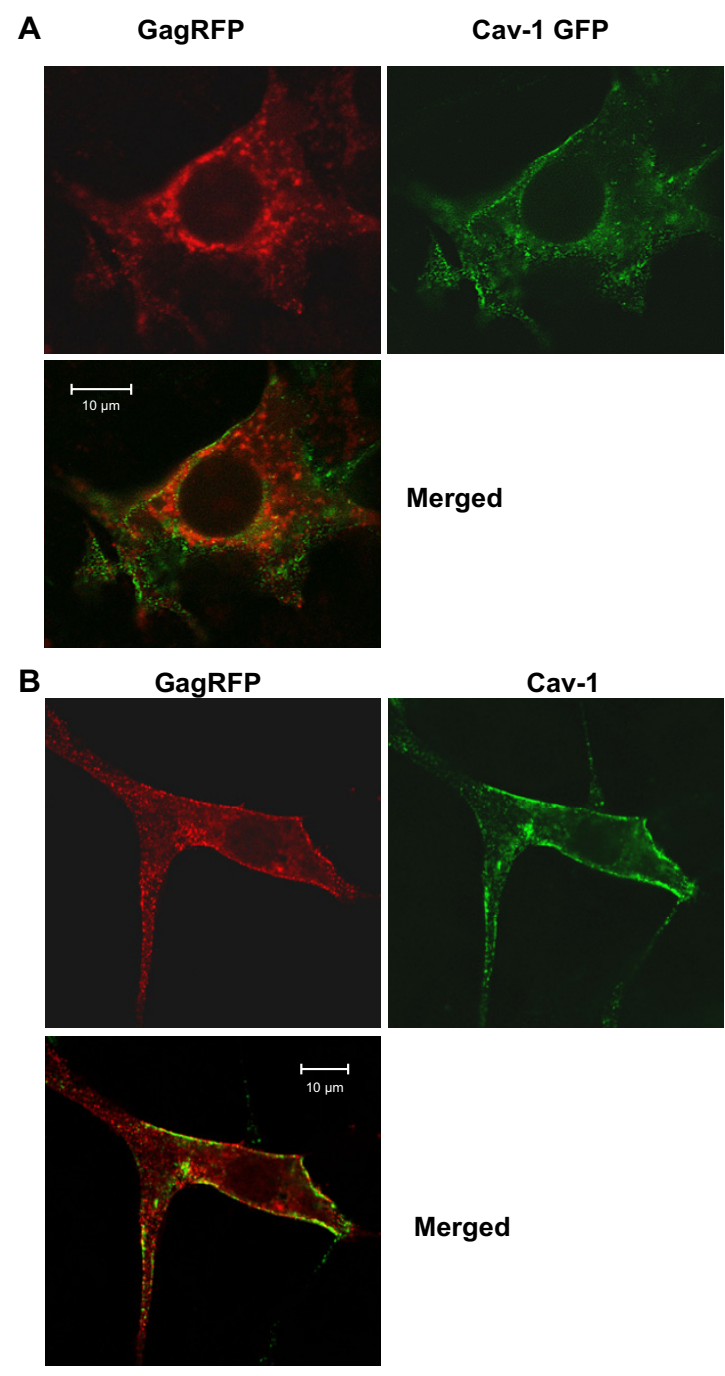

Figure 2

Colocalization studies using confocal microscopy. A. Caveolin- I-GFP and GagRFP fusion proteins colocalize in transiently transfected NIH 3T3 cells predominantly at the plasma membrane. NIH 3T3 cells were co-transfected with Gag-RFP and caveolin-I GFP plasmids, fixed $46 \mathrm{~h}$ later and analysed by confocal microscopy. B. Caveolin-I and GagRFP colocalization in NIH3T3 cells. NIH3T3 transfected with GagRFP plasmid were fixed $46 \mathrm{~h}$ after transfection and stained for immunofluorescence rabbit anti-caveolinlantibody followed by goat anti-rabbit-Alexa 488 conjugate

transfected cells and stained endogenous Cav-1 using an anti-Cav-1 antibody. Gag-RFP transfected NIH 3 T3 cells were fixed $46 \mathrm{~h}$ after transfection and immunostained with rabbit anti-Cav-1 antibody and goat anti rabbit Alexa 488. Confocal optical sections taken through the cell 
every $0.5 \mu \mathrm{m}$ revealed that Cav-1 as well as Gag-RFP are localized at the PM (see Additional file 2). Especially, GagRFP spots are scattered throughout the cytoplasm and perinuclear regions. However, both, Gag-RFP and Cav-1, co-localize in a dot-like pattern to a large extent at the PM (Fig. 2B, see Additional file 2). To improve visualization and to estimate the rate of co-localization 'correlation plots' were created. Therefore, the green and red channel were merged and co-localized pixels were highlighted in white. Approximately $40-70 \%$ of Gag-RFP and Cav-1 were co-localized at the PM (see Additional file 3). In addition, co-localization profiles revealed that, if not colocalized, Cav-1 and Gag spots at the PM are situated closely to another (see Additional file 4).

Taken together, our experiments reveal that Cav-1 and MLV Gag co-localize predominantly at the PM and to some degree in intracellular compartments. Moreover, MLV infection or the presence of other retroviral proteins does not influence the co-localization patterns.

\section{Gag-MA contains a putative caveolin-I binding domain, which is highly conserved among $\gamma$-retroviruses}

Cav-1 binds to a variety of cellular proteins via its caveolin scaffolding domain (CSD, aa82-101) [26]. Many of these binding partners play a role in cellular signaling. Two consensus domains for binding to Cav-1 (CBD) have been defined by phage display techniques using CSD as bait and a random peptide library [27]. Both consensus sequences were rich in aromatic residues and exhibited a

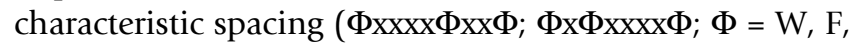
$\mathrm{Y})$. Interestingly, we identified a putative CBD motif in the MA of MoMLV and A-MLV Gag precursors (Table 1) [19]. Strikingly, the motif is highly conserved within most $\gamma$-retroviruses (Table 1) and is absent in Gag of other retroviruses.

\section{Caveolin-I interacts with Gag precursor of $M L V$}

Gag-Cav-1 co-localization, the presence of the putative CBD in MA of MLV and the high degree of conservation among $\gamma$-retroviruses motivated us to investigate whether the two proteins interact with each other. To determine whether MA-Cav-1 interactions occur in cells two types of binding experiments were carried out. In the first set coimmunoprecipitation experiments were performed using NIH 3T3 cells transfected with Gag-YFP or Gag-CFP expression plasmids. In these plasmids YFP or CFP variants have been fused to the C-terminus of MoMLV Gag [24]. Cells were lysed, Cav-1 was pulled down by an Cav1 antibody/proteinG and the precipitates were separated by SDS PAGE (10\%) and analyzed by Western Blot for their Gag-YFP or Gag-CFP content (Fig. 3). In samples transfected with Gag-CFP or Gag-YFP fusion plasmid (lane 2, lane 3) specific signals appeared at the expected size of $80 \mathrm{kD}$. No signals were detected in probes from mock-transfected NIH 3T3 cells (lane 4) or NIH 3T3 cells transfected with a GFP expression plasmid (lane 1), which excludes cross-reactions with GFP and Cav-1 during immunoprecipitation. Our data show that immunoprecipitation of Cav-1 results in recovery of a MLV Gag-Cav1 complex and strongly indicates that Cav-1 binds to MLV Gag.

In another set of experiments biotinylated peptides were used as baits for binding partners. The synthetic peptides encompassed the putative CBD of MA (MoMLV) (KKRRWVTFCSAEWPTFNVGW-K-Biotin) or a consensus CBD (RNVPPIFNDVYWIAFNVGAR-K-Biotin) [27]. After incubation with the cell lysates, complexes were bound on paramagnetic streptavidin beads. The eluate was separated via SDS PAGE and Western Blots were probed with polyclonal anti-Cav-1 antibody (Figure 4). Signals comigrating with the Cav-1 band of NIH $3 \mathrm{~T} 3$ extracts (positive control) appeared when either the biotinylated CBD of MA or a consensus CBD were incubated with the extract, but no signal cold be detected from NIH3T3 extract alone. In addition, two signals of minor intensity could be detected at $60 \mathrm{kD}$ and $80 \mathrm{kD}$ which may represent oligomeric forms of Cav-1.

The experiments demonstrate that a synthetic peptide comprising the putative CBD of MA of MoMLV pulls down Cav-1 as efficient as a consensus CBD peptide defined by phage display [27]. Taken together both series of experiments provide compelling evidence that MLV Gag directly interacts with Cav-1.

\section{CBD expression interferes with virus production}

We next performed experiments designed to investigate the biological significance of the Cav-1-Gag interaction. We reasoned that overexpression of fusion proteins containing the CBD of MA could block the interaction of Gag with endogenous Cav-1. To study the effect on virus formation expression plasmids were constructed encoding GFP fused to the CBD of MA or the consensus CBD peptide [27] as a positive control. Expression plasmids were transiently transfected into A-MLV producing NIH3T3 cells and the effect of CBD overexpression on infective virus production was determined by infectious titre assay (Figure 5). Results of three experiments show that virus production was reduced 5-10 fold upon CBD transfection. This suggests, that CBD overexpresssion competes with endogenous Cav-1 for binding to Gag and that Cav1 indeed plays a functional role in A-MLV production.

\section{A dominant negative caveolin-I mutant down-modulates virus production}

If endogenous Cav-1 is important for A-MLV production overexpression of Cav-1 or the interaction with a scaffolding-incompetent Cav-1 mutant should exert similar, neg- 
Table I: Putative caveolin-I binding domains in the matrix protein of the Gag precursor of $\gamma$-retroviruses

\begin{tabular}{|c|c|c|c|}
\hline Retrovirus & AA* & protein sequence $\dagger$ & Accession No \\
\hline Moloney MLV & 31 & KKRRWVTFCSAEWPTFNVGW & PIR:FOMVIM \\
\hline Sp $2 / 0$ xenotropic retrovirus & 31 & KKRRWVTFCSAEWPTFGVGW & EMBL : $\underline{\times 94150}$ \\
\hline Amphotropic MuLV 1313 & 31 & KKRRWVTFCSAEWPTFNVGW & GenBank:AF4II814.I \\
\hline Abelson MLV & 31 & KKRRWVTFCSAEWPTFNVGW & Swiss-Prot:P03333 \\
\hline AKV MLV & 31 & KKRRWVTFCSAEWPTFNVGW & Swiss-Prot: $\overline{\mathrm{P03336}}$ \\
\hline Rauscher MLV & 31 & RKRRWVTFCSAEWPTFNVGW & GenBank:NP 044737 \\
\hline Friend MLV & 31 & RKRRWVTFCSAEWPTFNVGW & Swiss-Prot:P26805 \\
\hline MAIDS related virus $(\mathrm{BXH}-2)$ & 31 & RKRRWVTFCSAEWPTFNVGW & GenBank:AAB47858.I \\
\hline MAIDS virus Duplan strain & 31 & KRRRWVTFCSVEWPSFNVGW & EMBL:XI4576 \\
\hline Gibbon ape leukemia virus SEATO & 31 & KKGKWQTFCSSEWPTFGVGW & Swiss-Prot:21416 \\
\hline Gibbon ape leukemia virus $X$ & 31 & RXGKWQTFCSSEWPTFGVRW & GenBank:AAC80263 \\
\hline Simian sarcoma virus & 31 & RKEKWQTFCSSEWPTFGVGW & PIR:FOMVGS \\
\hline Moloney murine sarcoma virus & 31 & KKRRWVTFCSAEWPTFNVGW & PIR:FOMVM \\
\hline Endogenous koala retrovirus & 31 & RKGKWQTFCSSEWPTFEVGW & GenBank:AAFI 5097 \\
\hline Porcine endogenous retrovirus & 31 & KKGPWQTFCASEWPTFDVGW & GenBank: $\overline{C A B 65341}$ \\
\hline Mus dunni endogeneous retrovirus (MDEV) & 31 & RKGPWQTFCASEWPTFGVGW & GenBank:AF053745 \\
\hline Mus musculus retrovirus (MmERV) & 31 & RKGPWQTFCTSEWPTFGVGW & GenBank:AC005743 \\
\hline Woolly monkey sarcoma virus & 31 & RKEKWQTFCSSEWPTFGVGW & Swiss-Prot :P03330 \\
\hline
\end{tabular}

AA: Amino acid position of the first residue in the depicted sequences is indicated. Bold, CBD motif identified from consensus CBD motifs [27] which were rich in aromatic residues and exhibited a characteristic spacing (

ative effects on virus yield. To evaluate the role of Cav-1 the effect of overexpressing wild-type (wt) Cav-1 or the dominant-negative Cav-1 mutant [28] on A-MLV virus production was investigated in infected NIH 3T3 cells which are known for their high Cav-1 content. Therefore, wt Cav-1 or Cav-1 Mut SD expression plasmid were transiently transfected into NIH 3T3 cells releasing a G418 resistant A-MLV and 48 hours after transfection viral titres were determined on indicator cells. A significant reduc-

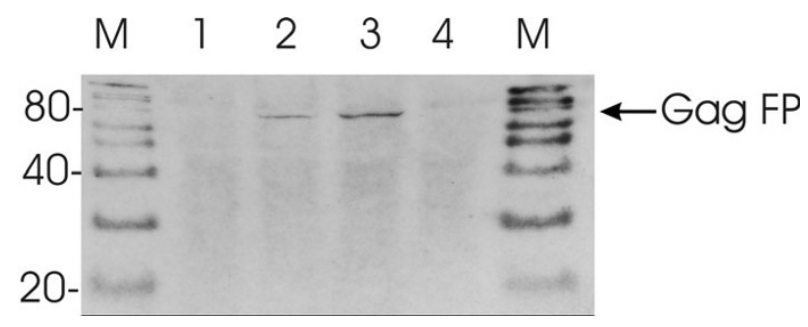

Figure 3

Co-immunoprecipitation of Cav-I. Lysates from or transfected (lane I-3) or mock-transfected (lane 4) NIH3T3 cells were treated with rabbit anti Cav-I followed by capture on paramagnetic protein $G$ microbeads ( $\mu$ column system, Miltenyi). Precipated proteins were separated by SDS-PAGE followed by Western Blot detection on PVDF membranes using an GFP antibody with GFP, YFP and CFP specifity. NIH3T3 lysates transfected with Lanel: GFP Plasmid; Lane 2: Gag-CFP; Lane 3: Gag-YFP Lane 4: mock; M Marker, molecular weight in $\mathrm{kDa}$ tion in virus titre was observed in Cav-1 Mut SD transfected cells when compared to mock-transfected cells (Figure 6). Interestingly, NIH 3T3 cells transfected with the wt Cav-1 construct exhibited a similar reduction in viral titre. These experiments suggest that inhibition of Cav-1 function as well as overexpression interfere with virus production and point to a discrete role of Cav-1 in late viral processes.

\section{Discussion}

We presented evidence that MLV Gag interacts with Cav-1 and that this interaction influences virus assembly and production. As another consequence of its interaction with Gag, Cav-1 is incorporated into A-MLV and MoMLV released from NIH 3T3 cells. Confocal fluorescence microscopy revealed that Cav- 1 and MLV Gag co-localize predominantly in punctate patterns at the PM and to lower extent in perinuclear regions of the cell. Sequence comparisons uncovered a Cav-1 binding domain in the matrix domain MA of the Gag precursor which is highly conserved among $\gamma$-retroviruses. Subsequent binding experiments using co-immunoprecipitation and a pulldown assay revealed that Cav-1 directly interacts with MLV-Gag. The interaction of Cav-1 with MA seems to play an important role in virus production, as overexpression of the CBD of MA considerably reduced the production of infectious virus in NIH 3T3 cells. Furthermore, overexpression of both, wt Cav-1 and a dominant-negative Cav1 mutant, in A-MLV releasing NIH 3T3 cells resulted in a considerable decrease of virus production. 


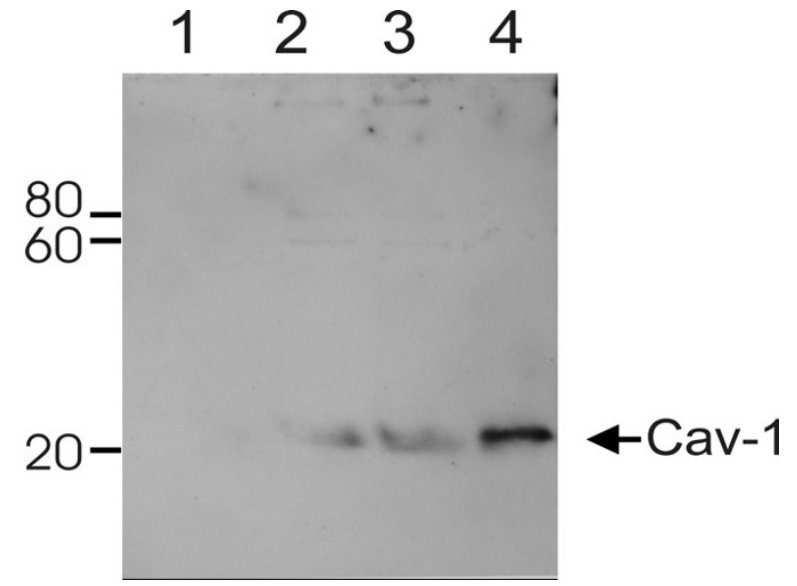

\section{Figure 4}

Pull down experiments using CBD peptides. $20 \mathrm{mM}$ biotinylated peptide encompassing either the putative binding domain within MA or a consensus binding motif were inoculated with $50 \mu \mathrm{l} \mathrm{NIH3T3}$ cell lysate for 90minutes (from $2 \mathrm{ml}$ lysate of semiconfluent T75 culture flask). Complexes were immobilized using $10 \mu \mathrm{l}$ streptavidin coated paramagnetic microbeads and $\mu$ column (Miltenyi). Washed samples were eluted and I $5 \mu \mathrm{l}$ of $80 \mu \mathrm{l}$ eluate were separated by SDSPAGE, blotted to PVDFmembrane and probed with anticaveolin-I antibody. lane I: NIH 3T3 lysate, no peptide added ; lane 2 : NIH3T3 lysate with biotinylated CBD-MA peptide; lane 3: NIH 3T3 incubated with biotinylated consensus CBD peptide; lane 3: positive control, NIH3T3 lysate, non-processed. Molecular weights are depicted $(\mathrm{kDa})$.

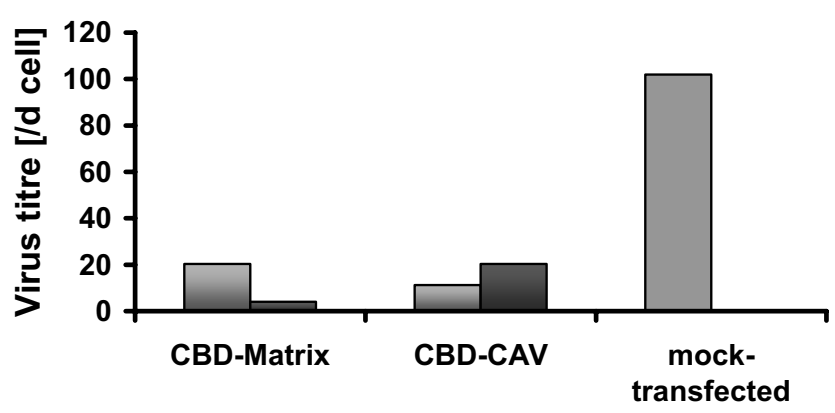

Competitor

\section{Figure 5}

Effect of CBD expression on MLV production. Expression plasmids carrying cloned caveolin-I binding domains were transfected into 4070A infected NIH3T3 cells and the effect on virus release was determined by infectious titer assay as described in Material and Methods. Competition experiments involved the putative CBD domain in MA of MLVs or a consensus CBD derived from display analysis [27].

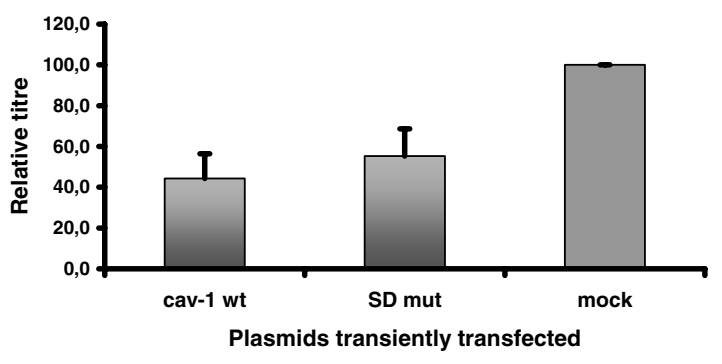

Figure 6

Influence of expression of cav-I wild-type and dominant-negative mutant cav-I on MLV-A titres in NIH3T3 cells. MLV-A infected NIH3T3 cells were transiently transfected with expression plasmids containing wt cav-I cDNA or a dominant-negative mutant carrying to mutations in the scaffolding domain [28]. Titres were determined from supernatants $48 \mathrm{~h}$ after transfection on indicator cells according to Spearman and Karber as described in Materials and Methods. Normalized values are shown. In each of three independent experiments mock-transfected NIH-MLV-A were used for normalization. Standard deviations are shown.

\section{The role of Cav-I in the MLV life cycle}

There are several lines of evidence that Cav-1 incorporation into virus and its interaction with Gag is of biological relevance. First, the CBD is highly conserved within $\gamma$-retroviruses. The strong selective pressure on preservation of the sequence argues for performance of a specific function within the viral life cycle. Second, perturbing the stochiometry of the interaction between Gag and Cav-1 by expression of wt Cav-1, a dominant negative Cav-1 mutant or fusion proteins carrying the CBD impaired viral life cycle and resulted in considerable decrease in viral yield. The inhibition is a specific process rather than an effect exhibited by interfering with cell viability or physiology. Obviously, perturbation interferes with late processes in viral replication. Third, further hints for the importance of the CBD of MA arise from deletion or linker scanning mutational analysis of the MA protein function performed earlier [5,29-31]. Strikingly, deletion or linker scanning mutation encompassing the region of the putative CBD resulted in dislocation of Gag and decrease in virus yield. For example, mutation of the tryptophan residues in a 'hydrophobic region', especially those which are part of (W43) or are close (W35, W50) to the putative CBD of MLV Gag (amino acids 38-46 of the Gag precursor) resulted in a dramatic loss in the production of infectious MoMLV and decrease in viral reverse transcriptase (RT) activity of released viruses. Mutations in residues 40, 44, 45 and 46 resulted in a 20 fold decrease 
in viral infectivity compared to the wt virus. As in this analysis the Gag localization pattern of tryptophan mutants differs considerably from wt Gag - the mutant Gag localizes exclusively in perinuclear regions in diffuse manner - the CBD tryptophan residues in MA identified in our investigation have been suggested to play an important role in Gag transport [5].

\section{Positioning of Gag to cellular membranes}

It is conceivable that Cav-1-Gag interaction is crucial for positioning MLV Gag at the PM, special PM domains like lipid rafts and/or the membrane of intracellular compartments or vesicles. If Cav-1 functions in that way it would function as a Gag receptor. The existence of a Gag receptor has been postulated, since the membrane insertion reaction is highly efficient and specific [32]. Retroviral Gag precursor proteins become anchored into the cytoplasmic leaflet of the PM via a dual motif consisting of amino terminal myristoylation and a cluster of basic residues [2$6,8]$. The dual motif is not expected to result in a very specific insertion, as myristoylated proteins are found in several compartments and acidic phospholipids, which interact with basic amino acids, are not restricted to the PM.

Interestingly, Cav-1 interaction with proteins substitutes for fatty acylation in certain cases and has been described to help in localization of proteins to lipid rafts. Partitioning of acyl side chains into liquid-ordered phase domains has been suggested as mechanism for targeting of proteins to lipid rafts [33]. However, although fatty acylation is necessary for membrane association of proteins in general, there is certain evidence that the normal requirement for acylation for localization in lipid rafts can be overcome by other molecules [34]. Studies with acyl-modified GFPs showed that N-terminal protein acylation only conferred localization to cholesterol and sphingolipidenriched membranes but not to lipid rafts or caveolae, suggesting that protein-protein interactions may be required for efficient raft association [34]. Also, acylated vesicular stomatitis virus (VSV) G protein and Rous sarcoma virus (RSV) Env were not associated with lipid raft [35]. Interestingly, it has been shown, that overexpression of a recombinant caveolin in intact cells is sufficient to functionally recruit a non-farnesylated Ras mutant onto membranes thereby overcoming the normal requirement for lipid modification of Ras. This suggests that caveolin may function as scaffolding protein to localize or sequester caveolin interacting proteins (e.g. wt Ras) within caveolin-rich microdomains of the PM [34]. Interestingly, caveolin is palmitoylated at 3 residues, but fatty acylation is not necessary for its caveolae localization [36]. Moreover, Pr60 Gag of murine AIDS virus lacking the myristoyl modification is not dispersed in the cytoplasma like MoMLV Pr65 Gag, but attaches loosely to the PM [37].
Overexpression of caveolin-1 in cells infected with a myristoylation minus MLV mutant and analysis of Gag localization and transport of mutants encompassing the MACBD motif and neighbourhood will elucidate more details on the importance of caveolin in Gag membrane attachment.

Presently, we do not know how many functions Cav-1 exerts in the MLV replication cycle. However, our results suggest that Cav-1 presumably is responsible for Gag localization within lipid rafts. According to our present understanding Cav-1 containing lipid rafts rather than caveolae itself seem to be most suitable for assembly and budding, as invagination, endocytosis and the compact coat of caveolae would exclude virus budding. Such an interpretation is supported by the characteristic localization patterns exhibited in profile analysis where co-localization and to some extent nearby localization of Gag and Cav-1 at the PM could be observed and a release process may be assumed when caveolae are formed upon Cav-1 oligomerization from preformed multimers. Furthermore, once localized, binding to Cav-1 may initiate oligomerization of further Gag molecules, leading to Gag clustering, a crucial oligomerization step in virus formation. Possibly, Cav-1 may also play a role in the transport of Gag to intracellular vesicles like MVBs or to the PM, either membrane bound, in its soluble form or asscociated with lipid droplets [38]. Finally, due to the fact that Cav-1 co-localizes with MLV-Env and Gag, it may serve as a Gag-Env bridging molecule. However, unlike in RSV or $\mathrm{HIV}$, there is little evidence for such a close linkage in the case of MLV [39,40].

Taken together, it is likely that Cav-1 functions to locate MLV Gag to the PM, and due to the co-localization of Env, Cav-1 and GM1 [16], a marker for lipid rafts, a role for Cav-1 in Gag positioning in lipid rafts is highly probable. It is tantalizing to speculate, that this will also hold for Gag of related retroviruses listed in Table 1 which contain the CBD motif.

Recently, Hovanessian et al. reported that HIV-gp41, the transmembrane subunit of the viral spike protein, also binds to caveolin-1 via a CBD motif located at position 622-633 of gp41 [41]. The CBD in gp41 is highly conserved within HIV-isolates and SIV lentivirus. However, the binding region was mapped to the lentiviral ectodomain of the transmembrane protein and the function of this putative interaction has not been revealed. Due to the external location of the CBD Cav-1 incorporation into virions has not been observed [41].

\section{Conclusion}

Taken together our data demonstrate that Cav-1 co-localizes with Gag of murine leukemia viruses at the PM and 
interacts with this precursor protein via a CBD in MA. As CBD competition or overexpression of a dominant-negative Cav-1 mutant affects virus production, Cav-1 plays distinct roles in virus assembly.

\section{Methods}

\section{Cells and viruses, cell culture}

NIH 3T3 cells (ATCC CRL-1658) and 293 cells (ATCC CRL 1573) were propagated in DMEM supplemented with antibiotics, glutamine and 10\% FCS. Cells were grown at $37^{\circ} \mathrm{C}, 5 \% \mathrm{CO}_{2}$ and $95 \%$ humidity.

\section{Plasmids, transfections and helper virus approach}

pMLV ampho and pMLVeco contain the complete genome of amphotropic MLV or ecotropic MLV cloned into pBluescript (Genethon, France, received via J.-C. Pages). pCaveolin-1-GFP contains canine cav-1 cDNA followed in frame by EGFP [21]. pCFPgag and pYFPgag are in frame fusion of MoMLV Gag with CFP or YFP [24]. pMLVgagRFP contains the MoMLV Gag ORF inserted into the BamHI/AgeI site of pmRFP-N1 (R.Tsien)[23]. pCSDMLV and pCSD-Consensus were created by insertion of an oligonucleotide coding for the putatitive CBD domain in $\mathrm{MA}$ and a oligonucleotide coding for the consensus CBD (see peptides), respectively, into the EcoRI site of pTarget (Promega). pLEIN contains a bicistronic MLV vector harboring EGFP and the neonmycin resistance gene (Clontech). pCav-WT contains a myc-tagged canine Cav-1 cDNA copy cloned into pCIS2 [28]. pCav-MUT contains point mutations (F92A V94A) in the scaffolding domain of canine cav-1 cDNA [28]. Transfections were performed using purified DNA (Quiagen kit) and the calcium-phosphate coprecitation method. MLV producing NIH3T3 cells resulted from calcium phosphate transfection of pMLVampho or pMLV eco, respectively, and subsequent infection of NIH3T3 with the respective replication competent MLV. G418 resistant viruses were created by cotransfection of pMLVs with pLEIN, which contains a bicistronic MLV vector harboring EGFP and the neomycin resistance gene (Clontech).

\section{Virus isolation}

MLVs were precipitated from cleared supernatants of MLV infected NIH 3T3 cells from three T75 flasks (2000 rpm, Heraeus Megafuge 1R) by centrifugation (3 h, $17000 \mathrm{rpm}$ Sorvall FAD-20C). Virus pellets were resuspended in TNE and purified by sucrose gradient centrifugation (25-40\% discontinuous, O/N $35000 \mathrm{rpm}$ ). Viruses banding at approx. 35\% sucrose were collected and precipitated at $40000 \mathrm{rpm}$ for $3 \mathrm{~h}$. All steps were carried out at $4^{\circ} \mathrm{C}$. Virus pellets were resuspended in $100 \mu \mathrm{l}$ TNE and stored at $20^{\circ} \mathrm{C}$.

\section{Virus titration}

Virus mediating G418 resistance were created by the helper approach and titrated on NIH 3T3 indicator cells (750 c per well, microtiter plate 96 well, 8 fold determination) according to the method of Spearman and Kaerber [42]. Serial dilutions of filtered supernatants (24 h production) were prepared and infection of indicator cells was performed in the presence of $8 \mu \mathrm{g} / \mathrm{ml}$ polybrene. Selective medium was applied $2 \mathrm{~d}$ after infection and clone forming units (cfu) were determined $10 \mathrm{~d}$ after infection by staining of cells with crystal violet.

\section{Peptide synthesis}

Biotin-labelled CBD peptides were synthesized by the group of Werner Tegge (Chemical Biology, GBF, Braunschweig, Germany). CBD-MA contained the putative CBD binding domain in MA (sequence AcRNVPPIFNDVYWIAFNVGAR-K-Biotin), CDB consensus a consensus Cav1 binding domain deviated from phage display experiments (sequence AcKKRWVTFCSAEWPTFNVGW-KBiotin) [27].

\section{Lysis of cells and viruses}

Cells or viruses were treated with lysis buffer (10 mM Tris pH7.5, $50 \mathrm{mM} \mathrm{NaCl}, 1 \%$ Triton X100, $60 \mathrm{mM}$ octylglucoside (Roche), $1 \mathrm{mM}$ aprotinin, $1 \mathrm{mM}$ leupeptin, $1 \mathrm{mM}$ PMSF) at $4^{\circ} \mathrm{C}$ for $30 \mathrm{~min}$ followed by centrifugation in an Eppendorf centrifuge at $15000 \mathrm{rpm}$. Cleared supernatants were processed further or stored at $-20^{\circ} \mathrm{C}$.

\section{Protein/protein binding assays}

Co-immunoprecipitation

Cell lysates were incubated with rabbit anti-caveolin-1 antibody $(1: 2000)$ at $4{ }^{\circ} \mathrm{C}$ for $1 \mathrm{~h}$ followed by incubation with $50 \mu \mathrm{l}$ Protein G-microbeads at $4^{\circ} \mathrm{C}$ for $1 \mathrm{~h}$ and subsequent application to prewashed $\mu$ columns (Miltenyi). After four washing steps (200 $\mu$ lysis buffer) bound proteins were eluted with $70 \mu \mathrm{l}$ sample buffer preheated to $95^{\circ} \mathrm{C}$.

\section{Pull-down assays}

Cell lysates were incubated with $20 \mu \mathrm{M}$ biotinylated CBD motif peptides at $4{ }^{\circ} \mathrm{C}$ for $90 \mathrm{~min}$ followed by treatment with $10 \mathrm{ml}$ streptavidin coated microbeads (Miltenyi Biotec). Lysate was applied on prewashed $\mu$ columns and after washing five times with $200 \mu \mathrm{l}$ lysis buffer the proteins were eluted with $70 \mu \mathrm{l}$ sample buffer preheated to $95^{\circ} \mathrm{C}$.

\section{SDS-PAGE and Western Blot analysis}

Proteins were separated by SDS-PAGE (12\%) at $100 \mathrm{~V}$ for $2 \mathrm{~h}$. Semidry blotting was used for subsequent protein transfer to PVDF membranes. After O/N blocking with Starting Block (PerBio), primary antibody (rabbit anticaveolin-1 or mouse anti-GFP diluted 1:2000 in starting 
block buffer containing $0.05 \%$ Tween 20) was applied at room temperature with constant shaking for $1 \mathrm{~h}$. Membranes were washed 3 times for $10 \mathrm{~min}$ in TBS/ $0.05 \%$ Tween 20 followed by incubation with the secondary antibody (goat anti-rabbit HRP $1: 100,000$ or goat-anti mouse HRP 1:1,000,000). After 5 additional washes membranes were incubated in luminal/enhancer solution (PerBio).

\section{Immunostaining and confocal immunofluorescence}

Cells were either fixed with $4 \%$ formaldehyde (cotransfections of fluorescent fusion proteins) or fixed/permeabilized with cold methanol/aceton (Gag-RFP, endogenous Cav-1) on coverslips. Blocking (2\% goat serum in PBS for $20 \mathrm{~min}$ ) was followed by incubation with the primary rabbit anti-caveolin-1 antibody (Transduction Laboratories, 1:300 diluted in PBS/2\% goat serum) for $1 \mathrm{~h}$. Excess antibody was removed by washing three times with PBS containing $0.02 \%$ TritonX-100. To detect the primary antibody, the samples were incubated with an Alexa Fluor 488-labeled goat-anti-rabbit secondary antibody (Molecular Probes, 1:300 dilution, Alexa Fluor $488 \mathrm{~F}\left(\mathrm{ab}^{\prime}\right)_{2}$ conjugate $\operatorname{IgG}(\mathrm{H}+\mathrm{L}))$. The coverslips were washed again and then mounted onto glass slides using fluorescent mounting medium (DAKO). Confocal imaging was performed with a Zeiss LSM 510META laser scanning microscope (inverted Axiovert $200 \mathrm{M}$ microscope) using a PlanApochromat $100 \times$ oil immersion objective (1.3 numeric apertures). EGFP or Alexa Fluor 488-labelled antigens were excited with an argon laser at $488 \mathrm{~nm}$, and emission was monitored using a 505-530 nm bandpass filter. For RFP visualization a HeNe laser at $543 \mathrm{~nm}$ and a 560-615 $\mathrm{nm}$ bandpass filter were used.

\section{Competition and inhibition experiments}

Plasmids pCSD-consensus or pCSD-MLV were stably introduced into A-MLV/pLEIN infected NIH3T3 cells by calcium phosphate precipitation. Virus titers were determined from pooled clones.

Plasmids pCav-wt or pCav-Mut were transiently introduced into A-MLV/pLEIN infected NIH3T3 cells by calcium phosphate precipitation. Virus titers were determined $2 \mathrm{~d}$ after transfection.

\section{Abbreviations}

CBD, caveolin binding domain; Cav-1, caveolin-1; MoMLV, murine leukaemia virus; A-MLV, amphotropic murine leukaemia virus; PM, plasma membrane; Env, envelope protein; Gag group-specific antigen; HIV, human immunodeficiency virus; GFP green fluorescent protein; YFP, yellow fluorescent protein; CFP, cyan fluorescent protein; RFP, red fluorescent protein; PMSF, phenyl methyl sulfonyl fluoride;

\section{Competing interests}

The author(s) declare that they have no competing interests.

\section{Authors' contributions}

MW and CB conceived the study. CB and MW performed the competition experiments. $\mathrm{CB}$ and $\mathrm{ZY}$ studied incorporation of cellular protein into virions. ZY performed the co-immunoprecipitations, pull down experiments and colocalization experiments. $\mathrm{MK}$ trained $\mathrm{ZY}$ in confocal microscopy and provided input for the fluorescenct colocalization experiments. MW performed the inhibition experiments, supervised all the experiments and drafted the manuscript. All authors read and approved the final manuscript.

\section{Additional material}

\section{Additional File 1}

Colocalization of Cav-1 and Gag RFP in transfected A-MLV infected NIH3T3 cells. A-MLV infected NIH3T3 were transfected with GagRFP plasmid, fixed $46 \mathrm{~h}$ after transfection and stained for immunofluorescence rabbit anti-caveolin-1 antibody followed by goat anti-rabbit-Alexa 488 conjugate.

Click here for file

[http://www.biomedcentral.com/content/supplementary/1743422X-3-73-S1.doc]

\section{Additional File 2}

Colocalization of Cav-1 and Gag RFP in transfected NIH3T3. Z-Stack images. NIH3T3 transfected with GagRFP plasmid were fixed $46 \mathrm{~h}$ after transfection and stained for immunofluorescence rabbit anti-caveolin1 antibody followed by goat anti-rabbit-Alexa 488 conjugate. Scanning by confocal microscopy from bottom to top, distance or $0.5 \mu \mathrm{m}$ each. Click here for file

[http://www.biomedcentral.com/content/supplementary/1743422X-3-73-S2.doc]

\section{Additional File 3}

Correlation plot and colocalization points of Cav-1 and Gag RFP fluorescence in NIH3T3 cells. The software merges the red (Ch3-T2) and green channel (Ch2-T1) and highlights colocalized pixels in white. Pixels are considered colocalized when their intensity is higher than the threshold of their channels (red label), which was defined by analysing the distribution frequency.

Click here for file

[http://www.biomedcentral.com/content/supplementary/1743422X-3-73-S3.doc]

\section{Additional File 4}

Profile analysis of Cav-1 and Gag RFP fluorescence in NIH3T3 cells. Profile was drawn by Zeiss software and depicts the intensity distribution (B) in the channels detecting GagRFP (red) and caveolin-1 (green) along the red arrow $(A)$.

Click here for file

[http://www.biomedcentral.com/content/supplementary/1743-

422X-3-73-S4.doc] 


\section{Acknowledgements}

We thank Michael Quon for providing us pCav-WT, pCav-MUT expression plasmids, Ari Helenius for the gift of $\mathrm{pC}$ Caveolin- I-GFP, Walter Mothes for PMLVgagRFP, Mary Collins for Gag-CFP and Gag-YFP fusion plasmids. We appreciate the technical help of Susanne Schertler and thank Werner Tegge (GBF) for peptide synthesis. The work was funded completely from institutional means.

\section{References}

I. Swanstrom R, Wills JW: Retroviruses. In Retroviruses Edited by: Coffin JM, Hughes SH, Varmus HE. Cold Spring Harbor Press, Cold Spring Harbor, N. Y.; 1997:263-334.

2. Rein A, McClure MR, Rice NR, Luftig RB, Schultz AM: Myristylation site in Pr65gag is essential for virus particle formation by Moloney murine leukemia virus. Proc Natl Acad Sci USA 1986, 83:7246-50.

3. Schultz AM, Rein A: Unmyristylated Moloney murine leukemia virus Pr65gag is excluded from virus assembly and maturation events. J Virol 1989, 63:2370-2373.

4. Zhou W, Parent LJ, Wills JW, Resh MD: Identification of a membrane-binding domain within the amino-terminal region of human immunodeficiency virus type I Gag protein which interacts with acidic phospholipids. / Virol 1994, 68:2556-2569.

5. Soneoka Y, Kingsman SM, Kingsman AJ: Mutagenesis analysis of the murine leukemia virus matrix protein: identification of regions important for membrane localization and intracellular transport. J Virol 1997, 71:5549-59.

6. Manrique ML, Gonzalez SA, Affranchino JL: Functional relationship between the matrix proteins of feline and simian immunodeficiency viruses. Virology 2004:157-67.

7. Granowitz C, Goff SP: Substitution mutations affecting a small region of the Moloney murine leukemia virus MA gag protein block assembly and release of virion particles. Virology 1994, 205:336-44.

8. Callahan EM, Wills JW: Repositioning basic residues in the $\mathbf{M}$ domain of the Rous sarcoma virus gag protein. J Virol 2000, 74: I I222-I I 229.

9. McCabe JB, Berthiaume LG: $\mathbf{N}$-terminal protein acylation confers localization to cholesterol, sphingolipid-enriched membranes but not to lipid rafts/caveolae. Mol Biol Cell 200I, | 2:360|-36|7.

10. Pornillos O, Garrus JE, Sundquist WI: Mechanisms of enveloped RNA virus budding. Trends Cell Biol 2002, 12:569-579.

II. Demirov DG, Freed EO: Retrovirus budding. Virus Res 2004, 106:87-102.

12. Segura-Morales C, Pescia C, Chatellard-Causse C, Sadoul R, Bertrand $\mathrm{E}$, Basyuk E: TsgIOI and Alix interact with murine leukemia virus Gag and cooperate with Nedd4 ubiquitin ligases during budding. J Biol Chem 2005, 280:27004-270I 2.

13. PickI WF, Pimentel-Muinos FX, Seed B: Lipid rafts and pseudotyping. J Virol 200I, 75:7175-7।83.

14. Sandrin V, Muriaux D, Darlix JL, Cosset FL: Intracellular trafficking of Gag and Env proteins and their interactions modulate pseudotyping of retroviruses. J Virol 2004, 78:7153-7I64.

15. Li M, Yang C, Tong S, Weidmann A, Compans RW: Palmitoylation of the murine leukemia virus envelope protein is critical for lipid raft association and surface expression. J Virol 2002, 76: I| $845-51 \mid 82$.

16. Beer C, Pedersen L, Wirth M: Amphotropic murine leukaemia virus envelope protein is assocated with cholesterol-rich microdomains. Virology 」 2005, 2:36.

17. Rothberg KG, Ying YS, Kolhouse JF, Kamen BA, Anderson RG: The glycophospholipid-linked folate receptor internalizes folate without entering the clathrin-coated pit endocytic pathway. J Cell Biol 1990, I I 0:637-649.

18. Liu P, Ying Y, Zhao Y, Mundy DI, Zhu M, Anderson RG: Chinese hamster ovary $\mathrm{K} 2$ cell lipid droplets appear to be metabolic organelles involved in membrane traffic. J Biol Chem 2004, 279:3787-3792.

19. Beer $C$, Wirth $M$ : The role of cholesterol rich domains and cellular proteins in mouse retrovirus assembly. Curr Top Virol 2004, 4:169-183.

20. Volonte D, Galbiati F, Lisanti MP: Visualization of caveolin-I, a caveolar marker protein, in living cells using green fluores- cent protein (GFP) chimeras. The subcellular distribution of caveolin-I is modulated by cell-cell contact. FEBS Lett 1999, 445:43I-439.

21. Pelkmans L, Kartenbeck J, Helenius A: Caveolar endocytosis of simian virus 40 reveals a new two-step vesicular-transport pathway to the ER. Nat Cell Biol 200I, 3:473-483.

22. Mundy DI, Machleidt T, Ying YS, Anderson RG, Bloom GS: Dual control of caveolar membrane traffic by microtubules and the actin cytoskeleton. J Cell Sci 2002, I I 5:4327-4339.

23. Sherer NM, Lehmann MJ, Jimenez-Soto LF, Ingmundson A, Horner SM, Cicchetti G, Allen PG, Pypaert M, Cunningham JM, Mothes W: Visualization of retroviral replication in living cells reveals budding into multivesicular bodies. Traffic 2003, 4:785-80I

24. Andrawiss M, Takeuchi Y, Hewlett L, Collins M: Murine leukemia virus particle assembly quantitated by fluorescence microscopy: role of Gag-Gag interactions and membrane association. J Virol 2003, 77: I 165I-1 1660.

25. Wang L, Connelly MA, Ostermeyer AG, Chen HH, Williams DL, Brown DA: Caveolin-I does not affect SR-BI-mediated cholesterol efflux or selective uptake of cholesteryl ester in two cell lines. J Lipid Res 2003, 44:807-815.

26. Li S, Couet J, Lisanti MP: Src tyrosine kinases, Galpha subunits, and $\mathbf{H}$-Ras share a common membrane-anchored scaffolding protein, caveolin. Caveolin binding negatively regulates the auto-activation of Src tyrosine kinases. J Biol Chem 1996, 27I:29|82-29190.

27. Couet J, Li S, Okamoto T, Ikezu T, Lisanti MP: Identification of peptide and protein ligands for the caveolin-scaffolding domain. Implications for the interaction of caveolin with caveolaeassociated proteins. J Biol Chem 1997, 272:6525-6533.

28. Nystrom FH, Chen H, Cong LN, Li Y, Quon MJ: Caveolin-I interacts with the insulin receptor and can differentially modulate insulin signaling in transfected Cos-7 cells and rat adipose cells. Mol Endocrinol 1999, 13:2013-24.

29. Jones TA, Blaug G, Hansen M, Barklis E: Assembly of gag-betagalactosidase proteins into retrovirus particles. J Virol 1990, 64:2265-79.

30. Hansen M, Jelinek L, Whiting S, Barklis E: Transport and assembly of gag proteins into Moloney murine leukemia virus. J Virol 1990, 64:5306-5316.

31. Hansen M, Jelinek L, Jones RS, Stegeman-Olsen J, Barklis E: Assembly and composition of intracellular particles formed by Moloney murine leukemia virus. J Virol 1993, 67:5163-5I74.

32. Suomalainen M, Hultenby K, Garoff H: Targeting of Moloney murine leukemia virus gag precursor to the site of virus budding. J Cell Biol 1996, I 35: I84I-I852

33. Melkonian KA, Ostermeyer AG, Chen JZ, Roth MG, Brown DA: Role of lipid modifications in targeting proteins to detergentresistant membrane rafts. Many raft proteins are acylated, while few are prenylated. J Biol Chem 1999, 274:3910-3917.

34. Song KS, Li SW, Okamoto T, Quilliam LA, Sargiacomo M, Lisanti MP: Co-purification and direct interaction of Ras with caveolin, an integral membrane protein of caveolae microdomains. Detergent-free purification of caveolae microdomains. J Biol Chem 1996, 271:9690-9697.

35. Li M, Vzorov AN, Weidmann A, Yenag C, Compans RW: Microbial subversion of host cells. In SGM symposium 62: Microbial subversion of host cells Cambridge University Press, Cambridge; 2003: I 28-144.

36. Uittenbogaard A, Smart E): Palmitoylation of caveolin-I is required for cholesterol binding, chaperone complex formation, and rapid transport of cholesterol to caveolae. I Biol Chem 2000, 275:25595-25599.

37. Huang M, Jolicoeur P: Myristylation of Pr60gag of the murine AIDS-defective virus is required to induce disease and notably for the expansion of its target cells. J Virol 1994, 68:5648-5655.

38. Martin S, Parton RG: Caveolin, cholesterol, and lipid bodies. Semin Cell Dev Biol 2005, 16:163-174.

39. Denesvre C, Carrington C, Corbin A, Takeuchi Y, Cosset FL, Schulz T, Sitbon M, Sonigo P: TM domain swapping of murine leukemia virus and human T-cell leukemia virus envelopes confers different infectious abilities despite similar incorporation into virions. J Virol 1996, 70:4380-4386.

40. Ragheb JA, Anderson WF: Uncoupled expression of Moloney murine leukemia virus envelope polypeptides SU and TM: a 
functional analysis of the role of TM domains in viral entry. J Virol 1994, 68:3207-3219.

41. Hovanessian AG, Briand JP, Said EA, Svab J, Ferris S, Dali H, Muller S, Desgranges $C$, Krust B: The caveolin-I binding domain of HIVI glycoprotein gp4 I is an efficient B cell epitope vaccine candidate against virus infection. Immunity 2004, 21:617-627.

42. Mayr A, Bachmann PA, Bibrack B, Wittmann G: Virologische Arbeitsmethoden Gustav Fischer, Jena; 1974:35-39.

Publish with Bio Med Central and every scientist can read your work free of charge

"BioMed Central will be the most significant development for disseminating the results of biomedical research in our lifetime. " Sir Paul Nurse, Cancer Research UK

Your research papers will be:

- available free of charge to the entire biomedical community

- peer reviewed and published immediately upon acceptance

- cited in PubMed and archived on PubMed Central

- yours - you keep the copyright

Submit your manuscript here:

http://www.biomedcentral.com/info/publishing_adv.asp
Biomedcentral 